\section{Is space time?}

Space and Time in the Microworld. By D. I. Blokhintsev. Pp. xiv +330 . (Reidel: Dordrecht and Boston, Mass., 1973.) Df. $110 ; \$ 39.50$.

Bотн relativity and quantum theory profoundly alter Newtonian concepts of space and time. Each theory introduces changes that are qualitatively different and their compatibility has been the topic of many debates. In special relativity, the individual process is still analysed in terms of point particles moving along trajectories in a manifold, although the interactions are always propagated with a bounded finite velocity. Quantum theory, on the other hand, only gives meaning to the individual process in terms of the ensemble and, furthermore, there seems to be an essential connectedness between interacting systems that are eventually spacially separated, giving rise to a correlation that seems to be non-local.

In the non-relativistic domain we know how to accommodate the quantum theory; the classical analogue of the phenomena can be successfully translated into the quantum algorithm, but once we move into the high energy region, the situation becomes somewhat confused. Does the essential connectedness of quantum theory imply that space-time descriptions are futile and that Lorentz invariance should be introduced through energy-momentum considerations alone or should we ask questions about possible modifications to space-time geometry? Indeed, the aim of Professor Blokhintsev's book is to present a critical analysis of this type of question.

He begins by developing the mathematical description starting from the Minkowski formalism of space-time, passing briefly through field theory, finally arriving at the S-matrix formalism which is to form the basis for the rest of the investigation. In fact, he goes as far as to claim that the S-matrix will be retained in all future theories, although the methods of constructing the matrix elements may undergo radical changes.

His main thesis then centres around the question of causality. In particular, he considers the consequences of replacing microscopic causality by a less restrictive form of macroscopic causality. This allows some form of acausality, or non-locality, in small space-time regions, while retaining causality in the large, The S-matrices constructed using either principle of causality differ only at energies that are much greater than the reciprocal of a fundamental length. Experiments show this to be less than $10^{-14} \mathrm{~cm}$ and the author speculates that it could be $10^{-16} \mathrm{~cm}$, the characteristic weak-interaction length. But the arguments are slender and it could equally well be at $10^{-33} \mathrm{~cm}$, the gravitational length, in which case further investigation might be discouraged. It has, however, often been suggested that the infinities in both classical and quantum field theories could be removed by changing the geometry in the small and the advantage of the principle of macroscopic causality is that it allows an exploration of various possibilities without leading to large-scale acausality. The final chapters are concerned with this question and discuss, in particular, the effects of discrete space-times, stochastic space-times and fluctuating geometries.

These final chapters left me feeling a little disappointed that more space had not been given to the question of generalised geometries. I find this a very fascinating field in which many questions are still unanswered. Indeed there is quite an extensive literature on the subject some of which is quite closely related to the topics discussed in the book but unfortunately no reference is made to this work. Apart from this small reservation, $I$ find the book very clearly written, and even the liberal use of mathematics has not been allowed to obscure the essential questions.

\section{B. J. HILeY}

\section{All sorts of skin}

The Integument. A Textbook of Skin Biology. By R. I. C. Spearman. Pp. vit 208. (Biological Structure and Function, vol. 3.) (Cambridge University: London, June 1973.) £4.20; \$12.95.

Because of the explosive developments taking place in science in general and biology in particular and because of the trend toward more and more specialisation, books which give a broad, comprehensive view of the subjects included under these headings have become a rarity. Thus, I began to read this book with some anticipation which was further whetted by the author's commitment to provide a bird's eye view of the whole subject of skin biology. As the author states in his preface, the comparative approach to the study of the outer covering of both invertebrates and vertebrates has not been attempted before; hence any book purporting to do so should be expected to fill a long-felt need. Unfortunately, The Integument fails to live up to these expectations and does little more than catalogue the various aspects of skin biology under various headings.

The book is divided into two parts, the first of which treats the comparative morphology of the integument of both invertebrates and vertebrates. Here
Spearman deals with the epidermal, dermal and pigmentary aspects of the integument of various phyla of invertebrates and of five classes of vertebrates (Pisces, Amphibia, Reptilia, Aves and Mammalia). This section is nicely illustrated with numerous line drawings which should help the beginner to understand the structural details of many of the skin appendages.

In the second part, the book deals with the comparative functions of skin, such as thermal regulation, chemical and neural control mechanisms, comparative synthetie processes, transport through the skin, immunity and development. The titles are provocative, but the cursory treatment leaves much to be desired. For example, in the chapter on the comparative synthetic process, only ten pages are devoted to such varied topics as genetic code, protein syntheses, elastin, resilin, collagen, and a host of others.

Brevity may be the soul of wit, but since wit is hardly the reason for undertaking a study such as this, the kind of one-sentence treatment accorded to important topies by Spearman while serving to whet the appetite does little to allay the hunger for substantial data.

Paul Parakkal

\section{Kidney disease}

Renal Histopathology: A Light Microscopy Study of Renal Disease. By Robert Meadows. Pp. xii +363 . (Oxford University: London and New York, December 1973.) $£ 14$.

Within the limits of the title Dr Meadows has written an interesting and very well illustrated book. It will be useful to all pathologists who examine renal biopsies and to the many physicians who seek to understand the structural changes in their patients. While agreeing with the author that most diagnoses can be achieved with the light microscope, fortified by knowledge obtained by others with ultrastructural and immunofluorescent methods $I$ feel it is a pity that there is not more reference to these in the text and some illustrations of the immunofluorescent results which after all are obtained with the light microscope.

The consideration of glomerular changes and glomerulonephritis is comprehensive and clear although the separation of the two leads to a certain amount of recapitulation. The section on the disappearance of glomeruli from the kidney is welcome in that it considers a difficult point which most authors skate over. Dr Meadows is not alone in attributing the clear description of what he calls the ischaemic obsolescent glomerulus to McManus (1950); it was in fact recognised by Russell (1929) by the 